\title{
Prise en charge de l'embolie pulmonaire grave en réanimation
}

\author{
Management of Massive Pulmonary Embolism in the Intensive Care Unit
}

\author{
Q. Philippot $\cdot$ A. Roche $\cdot$ C. Goyard $\cdot$ J. Pastré $\cdot$ B. Planquette $\cdot$ G. Meyer $\cdot$ O. Sanchez
}

Reçu le 21 février 2018; accepté le 11 mai 2018

(C) SRLF et Lavoisier SAS 2018

Résumé L'embolie pulmonaire (EP) grave, définie par la présence d'un état de choc, est à l'origine d'une mortalité importante. L'objectif de cette mise au point est de synthétiser les dernières avancées et recommandations concernant la prise en charge des formes graves d'EP. La stratification du risque individuel de mortalité précoce permet d'apporter une stratégie diagnostique et thérapeutique optimisée pour chaque patient. Le traitement symptomatique consiste essentiellement en la prise en charge de l'état de choc. L'anticoagulation curative par héparine non fractionnée est réservée aux patients hémodynamiquement instables. Chez ces patients à haut risque, la thrombolyse systémique diminue la mortalité et le risque de récidive d'EP. Chez les patients à risque intermédiaire élevé, la thrombolyse systémique à dose standard diminue le risque de choc secondaire mais sans impact sur la mortalité globale. La thrombolyse est donc réservée aux patients à risque intermédiaire élevé présentant secondairement un état de choc. L'embolectomie chirurgicale reste indiquée en cas de contre-indication absolue à la thrombolyse ou en cas d'échec de celle-ci. Le positionnement dans l'algorithme thérapeutique de l'assistance extracorporelle et des techniques percutanées de revascularisation reste à définir. Leurs indications doivent donc être discutées dans des centres experts après une concertation multidisciplinaire incluant pneumologues, cardiologues, réanimateurs, radiologues interventionnels et chirurgiens cardiaques.

Q. Philippot · A. Roche $\cdot$ C. Goyard · J. Pastré · B. Planquette

G. Meyer · O. Sanchez $(\bowtie)$

Service de pneumologie et soins intensifs,

hôpital européen Georges-Pompidou, AP-HP,

20, rue Leblanc, F-75015 Paris, France

e-mail : olivier.sanchez@aphp.fr

A. Roche $\cdot$ C. Goyard $\cdot$ B. Planquette $\cdot$ G. Meyer $\cdot$ O. Sanchez Université Paris-Descartes, France

15 rue de l'école de médecine, F-75006 Paris, France

B. Planquette $\cdot$ O. Sanchez

Inserm UMR-S 1140, Faculté de Pharmacie

4 avenue de l'Observatoire, F-75270 Paris Cedex 06, France
Mots clés Embolie pulmonaire · Anticoagulants · Thrombolyse

Abstract Major pulmonary embolism (PE), defined by the presence of shock, is a leading cause of mortality. The aim of this review is to summarize the latest advances and recommendations regarding the management of severe PE. The stratification of individual early mortality risk allows the optimization of the diagnostic and therapeutic approach for each patient. The symptomatic management is essentially focused on the hemodynamic support. Unfractioned heparin is indicated for hemodynamically unstable patients. In these high-risk patients, systemic thrombolysis decreases mortality and the risk of PE recurrence. In high-intermediate-risk patients, systemic thrombolysis given at current dosage decreases the risk of secondary hemodynamic decompensation but without effect on the overall mortality. Accordingly, thrombolytic therapy should be given to high-intermediate-risk patients in case of secondary hemodynamic worsening. Surgical embolectomy is still recommended in patients with absolute contraindication to thrombolysis and in case of thrombolysis failure. The place of extracorporeal assistance and percutaneous revascularization treatment in the therapeutic algorithm need to be better defined. Consequently, their indication should be decided in an expert center after a multidisciplinary discussion involving lung physician, cardiologist, interventional radiologist, and cardiac surgeon.

Keywords Pulmonary embolism · Anticoagulants · Thrombolytic therapy

La maladie thromboembolique veineuse (MTEV) est une maladie grave à l'origine d'une morbidité et d'une mortalité importantes. En 2004, 370000 décès ont été attribués à la MTEV dans six pays européens [1]. En France, les dernières estimations objectivent une incidence de l'embolie pulmonaire (EP) de 60 pour 100000 par an et un taux de mortalité variant de 4,7 à 19,2 \% [2]. Si l'EP n'est pas fatale d'emblée, sa gravité doit être évaluée à partir du risque individuel de décès à court terme (30 jours). Celui-ci est apprécié sur la 
présence de marqueurs pronostiques et non sur l'importance de l'obstruction anatomique vasculaire pulmonaire. L'objectif de cette mise au point est de détailler la prise en charge des EP graves correspondant aux formes d'EP compliquées d'un état de choc cardiogénique [3]. Compte tenu d'une certaine proximité de prise en charge, nous aborderons aussi la problématique des EP à risque intermédiaire élevé de mortalité précoce.

\section{Offrir la stratégie thérapeutique la plus adaptée au patient : stratification du risque de mortalité précoce}

\section{Identification des patients à haut risque de mortalité précoce}

Les deux principaux déterminants de la mortalité à court terme des patients atteints d'EP sont la tolérance hémodynamique et le terrain sous-jacent. Les registres objectivent une mortalité hospitalière de $25 \%$ chez les patients avec un état de choc, elle augmente à $62,5 \%$ chez ceux qui nécessitaient une réanimation [4]. La mortalité à trois mois des patients en état de choc est de 58,3\%. L'hypotension est un facteur de risque indépendant de mortalité [5]. Le risque de décès par EP est multiplié par 16,3 en cas d'état de choc (odds ratio [OR] : 16,3 ; IC $95 \%$ : [8,5-31,4]) [6]. Ces chiffres ont été confirmés dans une cohorte prospective multicentrique incluant 570 patients, qui rapportait une mortalité à 30 jours de $27 \%$ chez les patients en état de choc [7]. Chez ces patients, l'évaluation du retentissement ventriculaire droit de l'EP à l'aide de l'imagerie et/ou du dosage de biomarqueurs n'apporte aucune information pronostique supplémentaire [7]. L'état de choc, défini par une hypotension artérielle systolique en l'absence d'arythmie aiguë, d'hypovolémie ou de sepsis, est ainsi habituellement retenu dans la littérature pour définir les EP à haut risque (Fig. 1) [3]. Ces formes graves représentent environ $5 \%$ des EP [5].

\section{Identification des patients à faible risque de mortalité précoce}

Outre l'hypotension, les autres facteurs de risque de mortalité sont l'âge et l'existence d'une insuffisance cardiaque chronique, d'une insuffisance respiratoire chronique ou d'un cancer [5]. Le score PESI est un score de gravité élaboré rétrospectivement à partir d'éléments exclusivement cliniques et qui a fait l'objet d'une validation interne et de validations externes dans diverses cohortes [8] (Tableau 1). Ce score permet de prédire la mortalité précoce : de l'ordre de $2,5 \%$ pour les classes I et II, jusqu'à $24 \%$ pour la classe V. Une version simplifiée de ce score (sPESI) a été développée et validée dans des cohortes externes $[9,10]$. Les patients ayant un score sPESI à 0 ont un risque de mortalité à 30 jours de $1 \%$. Ce risque est de $11 \%$ chez ceux ayant un score sPESI supérieur ou égal à 1 [9]. Ces scores PESI ou sPESI possèdent une bonne sensibilité et une valeur prédictive négative pour exclure la survenue d'une complication à court terme mortelle ou non $[11,12]$. Un score PESI de classes I/II (score $\leq 85)$ ou un score $\mathrm{sPESI}=0$ permettent donc d'identifier les patients à faible risque de mortalité précoce (Fig. 1) [3].

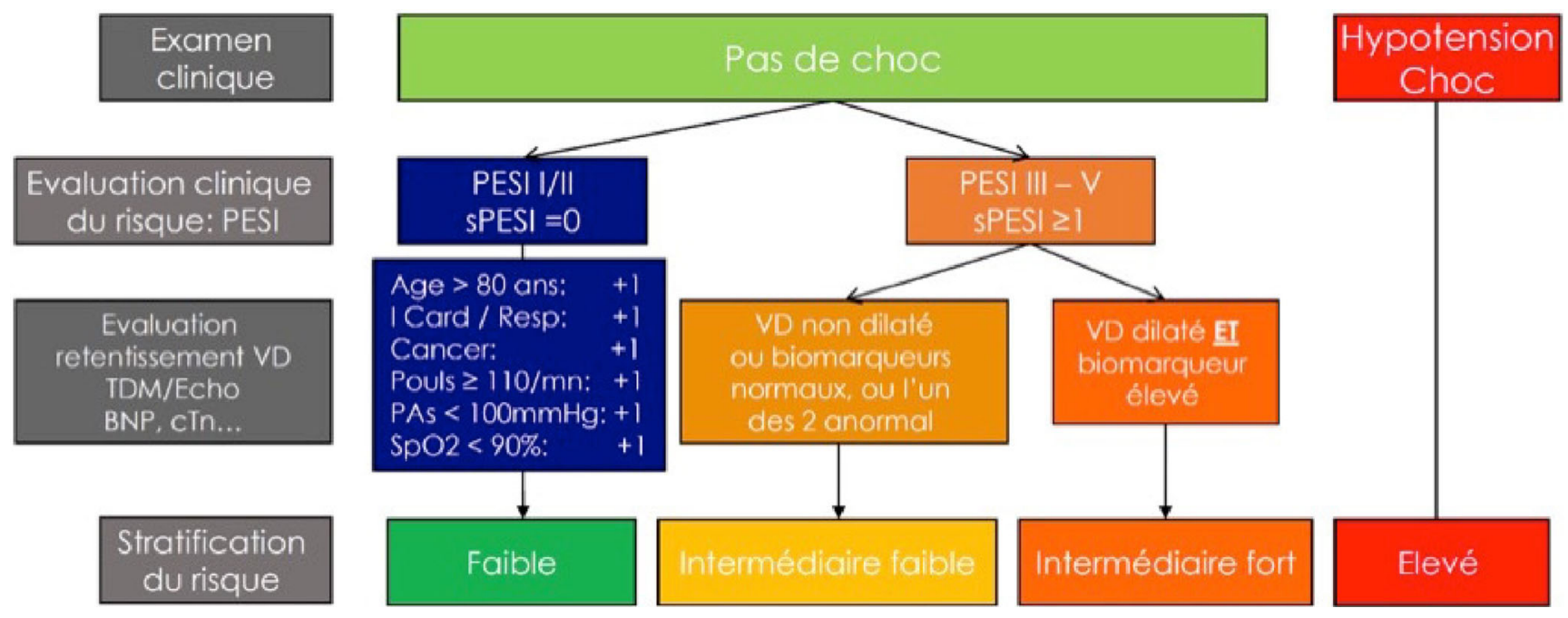

Fig. 1 Stratification du risque de décès précoce chez les patients avec une embolie pulmonaire PESI: pulmonary embolism severity index sPESI: score PESI simplifié I Card: insuffisance cardiaque I resp: insuffisance respiratoire PAs: pression artérielle systolique VD: ventricule droit 


\begin{tabular}{|c|c|c|}
\hline Paramètre & Version originale $[8]$ & Version simplifiée [9] \\
\hline Âge & Âge en années & 1 point si âge $>80$ ans \\
\hline Sexe masculin & +10 points & - \\
\hline Cancer & +30 points & 1 point \\
\hline Insuffisance cardiaque & +10 points & 1 point \\
\hline Insuffisance respiratoire & +10 points & \\
\hline Pouls $\geq 110 / \mathrm{min}$ & +20 points & 1 point \\
\hline Pression artérielle systolique $<100 \mathrm{mmHg}$ & +30 points & 1 point \\
\hline Fréquence respiratoire $>30 / \mathrm{min}$ & +20 points & - \\
\hline Température $<36{ }^{\circ} \mathrm{C}$ & +20 points & - \\
\hline Confusion & +60 points & - \\
\hline \multirow[t]{8}{*}{$\mathrm{SpO}_{2}<90 \%$ en air ambiant } & +20 points & 1 point \\
\hline & Classe de risque ; mortalité globale à j30 & \\
\hline & $\begin{array}{l}\text { Classe } I: \leq 65 \text { points } \\
(0-1,6 \%)\end{array}$ & $\begin{array}{l}\text { Faible risque : } 0 \text { point } \\
1,0 \%\end{array}$ \\
\hline & $\begin{array}{l}\text { Classe II : } 66-85 \text { points } \\
(1,7-3,5 \%)\end{array}$ & \\
\hline & $\begin{array}{l}\text { Classe III : } 86-105 \text { points } \\
(3,2-7,1 \%)\end{array}$ & $\begin{array}{l}\text { Autres }: \geq 1 \text { point } \\
10,9 \%\end{array}$ \\
\hline & Classe IV : $106-125$ points & \\
\hline & $(4,0-11,4 \%)$ & \\
\hline & Classe V : $>125$ points & \\
\hline
\end{tabular}

\section{Identification des patients à risque intermédiaire}

Les patients stables hémodynamiquement n'appartenant pas au groupe à faible risque sont considérés à risque intermédiaire de mortalité et de complications. Chez ces patients, le retentissement ventriculaire droit, évalué par l'imagerie (échocardiographie ou scanner) et/ou le dosage de biomarqueurs (BNP, troponine...), possède un rôle pronostique additionnel [7,13-15]. Pris isolément, chacun de ces paramètres possède une bonne valeur prédictive négative (>95\%) pour prédire la survenue d'une complication précoce mortelle ou non [3]. En d'autres termes, en l'absence de dysfonction ventriculaire droite ou d'une élévation de l'un des biomarqueurs, un patient avec une EP dépourvue d'instabilité hémodynamique est à faible risque de complications. Toutefois, la valeur prédictive positive de ces marqueurs est très médiocre $(\approx 10 \%)$ [3]. L'utilisation combinée de ces marqueurs dans des scores pronostiques pourrait s'avérer intéressante pour identifier des patients à risque intermédiaire de mortalité.

Les recommandations ESC de 2014 définissent les patients à risque intermédiaire par l'absence d'état de choc et un score PESI supérieur ou égal à 86 ou un sPESI supérieur ou égal à 1 (Fig. 1) [3]. Les patients à risque intermédiaire faible sont distingués de ceux à risque intermédiaire élevé en évaluant le retentissement ventriculaire droit [3].
Les patients à risque intermédiaire faible sont définis par un SPESI supérieur à 0 , associé ou non à un ventricule droit dilaté ou à un biomarqueur élevé [3]. Les patients à risque intermédiaire élevé sont définis par un sPESI supérieur à 0 , une dilatation ventriculaire droite $(\mathrm{VD} / \mathrm{VG} \geq 0,9)$ et un biomarqueur élevé [3]. Ces patients ont un risque de décès à 30 jours de $8 \%$ [16] et de décès ou de complications de $11 \%$ [17].

\section{Anticoagulation}

Toutes les études ayant évalué la fibrinolyse au cours de l'EP grave ont été réalisées avec de l'héparine non fractionnée (HNF). L'HNF administrée par voie intraveineuse continue reste donc la modalité d'anticoagulation initiale à privilégier chez les patients à haut risque en raison d'une plus grande maniabilité [3]. Les patients avec une EP grave et a fortiori ceux recevant un traitement fibrinolytique n'étaient pas inclus dans les essais thérapeutiques ayant évalué les héparines de bas poids moléculaire (HBPM), le fondaparinux et les anticoagulants oraux directs. Quelques données rétrospectives ou prospectives ayant inclus de petits effectifs rapportent une efficacité et une sécurité satisfaisantes des HBPM chez des patients traités par fibrinolytiques pour une EP $[18,19]$. Chez les patients sans état de choc avec 
une EP à risque intermédiaire élevé, l'utilisation d'une HBPM semble être la modalité d'anticoagulation initiale à privilégier pour les 24-48 premières heures d'hospitalisation. En l'absence de traitement fibrinolytique et/ou après amélioration clinique, la poursuite de l'HBPM relayée par un antivitamine $\mathrm{K}$ ou l'utilisation d'un anticoagulant oral direct (rivaroxaban ou apixaban) sont deux options envisageables.

\section{Stratégies de revascularisations dans l'embolie pulmonaire}

\section{Thrombolyse systémique}

Dix-huit études randomisées ayant inclus un total de 2197 patients et ayant comparé différents agents thrombolytiques à un traitement anticoagulant par HNF ont été publiées [20]. Ces données ont été compilées dans cinq méta-analyses [21-25]. L'interprétation des données issues des essais randomisés nécessite de prendre en considération un certain nombre de limitations. La sévérité de l'EP, le critère de jugement principal, la nature de l'agent thrombolytique utilisé, sa posologie, sa durée d'administration varient en effet d'une étude à l'autre. En outre, les critères cliniques d'instabilité hémodynamique, de choc ou d'hémorragie ne sont pas définis de façon standardisée.

Une amélioration plus rapide de l'obstruction vasculaire pulmonaire, de la pression artérielle pulmonaire et des résis- tances pulmonaires ainsi que de la dilatation ventriculaire droite échographique est observée sous traitement thrombolytique comparativement à l'HNF. Une réduction de la mortalité et des récidives emboliques est rapportée dans les données issues des méta-analyses dans les études qui ont inclus des patients avec EP à haut risque définie par un état de choc ou une hypotension artérielle systémique. Ce bénéfice est grevé d'un risque accru d'hémorragie sévère $(9,2 \%)$, en particulier intracrânienne $(1,5 \%)[21,22]$. Il convient toutefois de noter que la majorité de ces données est issue d'études anciennes avec des procédures diagnostiques invasives et des doses de thrombolytiques élevées. Les sociétés savantes recommandent donc le recours à la fibrinolyse chez les patients avec une EP grave définie par la présence d'un état de choc en l'absence de contre-indication (Tableau 2).

Chez les patients à risque intermédiaire élevé, la thrombolyse a été évaluée dans l'étude PEITHO qui a randomisé 1006 patients avec une pression artérielle normale, une dilatation du ventricule droit et une élévation de la troponinémie pour recevoir soit de l'héparine et du ténectéplase, soit de l'héparine seule. Le taux de mortalité ou de survenue secondaire d'un état de choc à sept jours (critère de jugement principal) était significativement réduit dans le groupe ténectéplase $(1,6$ vs $5,0 \%, p=0,002)$. Les taux de saignements majeurs extracrâniens $(6,3$ vs $1,2 \%, p<0,001)$ et intracrâniens $(2,0$ vs $0,2 \%, p=0,003)$ étaient significativement plus élevés, sans impact sur la mortalité $(1,2$ vs $1,8 \%, p=0,43)$ [26]. Le suivi à deux ans de 709 patients inclus dans l'essai PEITHO n'a pas objectivé de bénéfice de la thrombolyse sur

Tableau 2 Agents thrombolytiques, posologies et contre-indications

\begin{tabular}{|c|c|c|}
\hline Molécule & Régime & Contre-indications à la fibrinolyse \\
\hline \multirow[t]{3}{*}{ Streptokinase } & $250000 \mathrm{U}$ en dose de charge sur $30 \mathrm{~min}$, suivi & Absolues \\
\hline & de $100000 \mathrm{U} / \mathrm{h}$ sur $12-24 \mathrm{~h}$ & $\overline{\text { AVC hémorragique ou de nature indéterminée }}$ \\
\hline & Régime accéléré : 1,5 million $\mathrm{U}$ sur $2 \mathrm{~h}$ & AVC ischémique datant de moins de six mois \\
\hline \multirow[t]{3}{*}{ Urokinase } & $4400 \mathrm{U} / \mathrm{kg}$ de poids corporel en dose de charge & Traumatisme SNC ou néoplasme \\
\hline & sur $10 \mathrm{~min}$, suivi de $4400 \mathrm{U} / \mathrm{kg}$ par heure sur $12-24 \mathrm{~h}$ & Traumatisme majeur, chirurgie, ou traumatisme \\
\hline & Régime accéléré : 3 millions $U$ sur $2 \mathrm{~h}$ & crânien dans les trois semaines précédentes \\
\hline \multirow[t]{13}{*}{ Altéplase } & $100 \mathrm{mg}$ sur $2 \mathrm{~h}$ & Hémorragie gastro-intestinale au cours du dernier \\
\hline & Régime accéléré : $0,6 \mathrm{mg} / \mathrm{kg}$ sur $15 \mathrm{~min}$ & mois \\
\hline & & Risque hémorragique documenté \\
\hline & & $\underline{\text { Relatives }}$ \\
\hline & & $\overline{\text { AIT au cours des six derniers mois }}$ \\
\hline & & Traitement par anticoagulant oral en cours \\
\hline & & Grossesse ou $<1$ semaine post-partum \\
\hline & & Impossibilité de compression au point de ponction \\
\hline & & Manœuvres de ressuscitation \\
\hline & & Hypertension réfractaire $(\mathrm{PAs}>180 \mathrm{mmHg})$ \\
\hline & & Insuffisance hépatique sévère \\
\hline & & Endocardite infectieuse \\
\hline & & Ulcère gastrique évolutif \\
\hline
\end{tabular}


la mortalité à long terme, la dyspnée et la dysfonction ventriculaire droite résiduelle [27]. L'utilisation des thrombolytiques n'est donc pas recommandée de manière systématique chez les patients avec une EP à risque intermédiaire élevé, mais doit être envisagée en cas d'apparition de signe de choc [3]. Ces patients doivent donc être admis en unité de soins continus ou intensifs afin de proposer une thrombolyse secondaire dès l'apparition des signes de choc. Cette dégradation hémodynamique concernerait $5 \%$ des patients et interviendrait dans un délai moyen de 1,8 jour après l'admission [26]. Dans une méta-analyse, la thrombolyse, dans le sous-groupe de patients à risque intermédiaire, était associée à une baisse significative de la mortalité liée à l'EP mais non significative de la mortalité globale. La thrombolyse n'était pas associée à une baisse des récurrences d'EP, mais était en revanche associée à une augmentation des hémorragies majeures $(\mathrm{OR}=2,91 ; p<0,001)$ et des hémorragies intracrâniennes ou fatales $(\mathrm{OR}=3,18 ; p=0,008)$ [22]. Cette augmentation du risque d'hémorragies intracrâniennes ou fatales explique probablement l'absence de bénéfice de la thrombolyse sur la mortalité globale chez ces patients.

Ces résultats posent le rationnel pour l'évaluation de doses plus faibles de fibrinolytiques chez les patients avec une EP à risque intermédiaire élevé. Deux études prospectives ayant inclus de faibles effectifs suggèrent que la thrombolyse à dose réduite pourrait avoir des effets hémodynamiques bénéfiques sans majorer le risque de saignement $[19,28]$. Ces résultats nécessitent d'être confirmés dans des essais de plus large envergure évaluant des critères de jugement cliniques pertinents.

\section{Traitements percutanés dirigés par cathéter}

Ces traitements regroupent entre autres : la fragmentation mécanique du thrombus par le cathéter, la fragmentation rhéologique, la thromboaspiration, la thrombectomie rotationnelle ainsi que la thrombolyse dirigée par cathéter. Les données concernant l'évaluation de la reperfusion instrumentale percutanée dans le traitement de l'EP aiguë sont limitées. Une seule étude randomisée a été conduite, qui a inclus 59 patients avec EP à risque intermédiaire définie par la présence d'une dysfonction ventriculaire droite échocardiographique [29]. Trente-cinq études ont été publiées, regroupant 1253 patients et ayant fait l'objet de quatre méta-analyses ou revues systématiques [30-33].

L'essai ULTIMA est le seul essai randomisé étudiant une technique de revascularisation percutanée. Cinquante-neuf patients présentant une EP avec dysfonction ventriculaire droite sans instabilité hémodynamique étaient randomisés. Par comparaison à l'héparine seule, la thrombolyse accélérée par les ultrasons et dirigée par cathéter réduisait de manière significative le rapport VD/VG 24 heures après la randomisation sans majoration du risque hémorragique. Deux regis- tres prospectifs, multicentriques, (SEATTLE II et PERFECT) ont confirmé l'amélioration significative de la fonction ventriculaire droite ou des pressions artérielles pulmonaires chez les patients avec EP à risque intermédiaire ou élevé $[34,35]$. Aucune hémorragie cérébrale n'a été observée dans ces deux registres ayant regroupé au total 251 patients, mais un taux de complication hémorragique grave de $10 \% \mathrm{a}$ été rapporté dans le registre SEATTLE II [35].

L'interprétation de ces résultats doit prendre en compte le caractère limité des données, l'absence d'étude comparative avec un traitement thrombolytique intraveineux et l'absence d'informations sur l'évolution clinique au long terme des patients. Des biais de publication ne sont pas à exclure, compte tenu des taux très faibles de complications hémorragiques graves rapportés dans la littérature chez des patients ayant fait l'objet d'un abord veineux fémoral et d'un traitement thrombolytique. En outre, il convient de prendre en considération qu'en plus d'infrastructures appropriées, une expertise médicale locale est nécessaire à la réalisation de ce type d'intervention. Enfin, aucun des cathéters actuellement disponibles sur le marché ne fait l'objet d'un remboursement en France.

Les recommandations européennes de 2014 proposent d'envisager les traitements percutanés dirigés par cathéter comme une alternative à l'embolectomie chirurgicale si les ressources et l'expertise sont disponibles dans le centre de prise en charge [3].

\section{Embolectomie chirurgicale}

L'embolectomie chirurgicale reste indiquée chez les patients ayant une contre-indication absolue à la thrombolyse ou en cas d'échec de celle-ci. La présence d'un thrombus intracavitaire en échocardiographie, en particulier lorsqu'il est enclavé dans un foramen ovale perméable, peut également constituer une indication chirurgicale [36,37].

Sur le plan technique, l'embolectomie chirurgicale est réalisée sous circulation extracorporelle, sans clampage aortique ni cardioplégie, et doit être pratiquée par une équipe expérimentée.

On ne dispose d'aucun essai randomisé comparant embolectomie contre fibrinolyse. En l'absence d'instabilité hémodynamique préalable, la mortalité opératoire des EP est de l'ordre de $16 \%$ [38-41]. La survie, la symptomatologie fonctionnelle et la qualité de vie se révèlent également satisfaisantes au long cours $[42,43]$. La réalisation d'une embolectomie au décours d'une thrombolyse majore le risque hémorragique, mais ne constitue pas une contre-indication absolue à la chirurgie $[44,45]$. Elle est associée à une évolution hospitalière plus favorable qu'une nouvelle thrombolyse en cas d'échec de la première [44]. 


\section{Prise en charge symptomatique de l'état de choc au cours de l'embolie pulmonaire}

La dysfonction ventriculaire droite est la principale cause de mortalité chez les patients présentant une EP grave. Les recommandations européennes proposent :

- une expansion volémique prudente ;

- l'utilisation d'inotropes pour améliorer la fonction ventriculaire droite lorsque la pression artérielle est basse ;

- si nécessaire de ventiler les patients avec un volume courant et une pression de plateau faible.

\section{Expansion volémique}

L'utilisation de l'expansion volémique repose sur l'application de la loi de Frank-Starling au ventricule droit. Dans une étude observationnelle incluant 13 patients présentant une EP grave, il a été démontré qu'un remplissage modéré (500 ml) augmentait l'index cardiaque [46]. L'interdépendance ventriculaire peut cependant s'opposer au bénéfice attendu : la compression des cavités gauches par les cavités droites dilatées peut faire chuter la précharge ventriculaire gauche et ainsi aggraver l'état hémodynamique. L'augmentation des pressions ventriculaires droites entraîne par ailleurs une ischémie myocardique par baisse du gradient de perfusion coronaire [47]. L'utilisation de l'expansion volémique dans l'EP est donc controversée. Dans une étude rétrospective incluant 70 patients présentant une EP de gravité intermédiaire, on observait une diminution significative de l'index de choc à 24 heures chez les patients traités par furosémide contrairement à ceux traités par remplissage. Il n'était pas observé de différence de mortalité ni de choc secondaire entre les deux groupes [48]. Le bénéfice des diurétiques dans la prise en charge de l'EP de gravité intermédiaire est actuellement étudié dans un essai randomisé prospectif multicentrique (DIPER NCT02268903).

\section{Inotropes}

La dobutamine est historiquement considérée comme l'inotrope de référence en cas de choc ou d'hypotension. Dans une série de dix patients, Jardin et al. rapportaient une augmentation de l'index cardiaque et une baisse des résistances vasculaires pulmonaires après une perfusion de 30 minutes de dobutamine. La noradrénaline semble efficace pour améliorer la fonction ventriculaire droite et le débit cardiaque en cas de choc [49]. Dans un modèle canin d'EP grave, une perfusion de noradrénaline augmente le débit cardiaque contrairement à l'expansion volémique [50]. Il n'existe actuellement aucune étude comparant l'utilisation de la noradrénaline par rapport à la dobutamine dans la stabilisation hémodynamique des patients présen- tant une EP grave. L'utilisation de lévosimendan pourrait améliorer le couplage entre la fonction ventriculaire droite et le réseau artériel pulmonaire en associant une vasodilatation pulmonaire et une augmentation de la contraction ventriculaire droite [51].

\section{Ventilation mécanique et monoxyde d'azote}

L'utilisation de la ventilation mécanique comporte un risque de majoration de la défaillance hémodynamique [52]. Les indications de la ventilation mécanique sont rares au cours de l'EP grave. Elles concernent les patients en arrêt cardiaque et ceux présentant des troubles de la vigilance. En cas de recours à la ventilation mécanique, il est recommandé d'utiliser un faible volume courant et d'éviter la pression expiratoire positive afin de limiter les effets délétères hémodynamiques liés à l'augmentation de pression intrathoracique.

L'utilisation de monoxyde d'azote en inhalation a montré un bénéfice hémodynamique chez quelques patients [53].

\section{Assistance circulatoire}

En cas de persistance de l'état de choc malgré un traitement symptomatique optimal, l'assistance circulatoire extracorporelle veinoartérielle (ECMO) semble apporter un bénéfice. Elle permet de rapidement décharger le ventricule droit, d'améliorer le débit cardiaque et de restaurer une perfusion périphérique efficace. Elle est indiquée chez les patients en état de choc réfractaire et/ou avec de multiples dysfonctions d'organe. Elle nécessite toutefois une équipe médicochirurgicale entraînée disponible 24 heures/24. Une étude descriptive monocentrique, incluant 17 patients pris en charge pour une EP grave (dont 15 patients en arrêt cardiaque) traités par ECMO, objectivait une survie de $47 \%$ à 90 jours [54]. Chez les huit patients traités uniquement par ECMO et anticoagulation, l'ECMO était sevrée en quatre jours en moyenne avec une excellente survie. Les auteurs suggèrent donc la possibilité de surseoir aux traitements de reperfusion artérielle pulmonaire (fibrinolyse, thrombectomie chirurgicale ou percutanée) chez ces patients. Toutefois, l'analyse des articles rapportant l'utilisation de l'ECMO chez les patients avec une EP grave montre que la quasi-totalité des patients avait reçu un traitement de reperfusion artérielle pulmonaire avant ou peu de temps après la pose de l'ECMO. De plus, toutes ces études ne rapportaient pas systématiquement les complications liées à l'ECMO. Il est donc difficile d'évaluer l'efficacité de l'ECMO en association avec l'anticoagulation comme seul traitement chez des patients avec une EP grave. Néanmoins, ces études rapportaient une survie " acceptable », variant de 25 à $100 \%$, compte tenu du niveau de gravité des patients (40 à $100 \%$ d'entre eux avaient présenté un arrêt cardiaque). En résumé, ces 
Tableau 3 Synthèse de la prise en charge de l'EP grave

\begin{tabular}{|c|c|c|}
\hline EP à risque intermédiaire élevé & \multicolumn{2}{|l|}{ EP à haut risque } \\
\hline PESI de classe III ou I-sPESI $\geq 1$ & \multirow{2}{*}{\multicolumn{2}{|c|}{$\begin{array}{l}\text { PAs }<90 \mathrm{mmHg} \text { ou chute }>40 \mathrm{mmHg} \text { par rapport à la PAs de base pendant } \\
\text { au moins } 15 \text { minutes }\end{array}$}} \\
\hline Absence d'hypotension & & \\
\hline \multicolumn{3}{|l|}{ DVD $^{\mathrm{a}}$ et biomarqueurs élevés } \\
\hline \multicolumn{3}{|c|}{ Hospitalisation en soins intensifs ou en réanimation } \\
\hline Anticoagulation par HBPM & \multicolumn{2}{|c|}{ Anticoagulation par héparine non fractionnée } \\
\hline \multirow[t]{2}{*}{ Prévenir la dégradation hémodynamique } & \multicolumn{2}{|l|}{ Stabilisation hémodynamique } \\
\hline & \multicolumn{2}{|l|}{ Objectif : PAm $>65 \mathrm{mmHg}$} \\
\hline Diurétiques $^{\mathrm{a}}$ & Remplissage modéré (500 ml) & ECMO veinoartérielle ${ }^{\mathrm{a}}$ \\
\hline Thrombolyse à demi-dose ${ }^{\mathrm{a}}$ & Dobutamine ou Noradrénaline & NO inhalé \\
\hline & & Lévosimendan $^{\mathrm{a}}$ \\
\hline \multirow[t]{3}{*}{ En cas de dégradation hémodynamique $\rightarrow$} & \multicolumn{2}{|l|}{ Reperfusion } \\
\hline & Thrombolyse & Embolectomie percutanée ${ }^{\mathrm{a}}$ \\
\hline & \multicolumn{2}{|l|}{ Embolectomie chirurgicale } \\
\hline
\end{tabular}

données suggèrent que l'ECMO est un support hémodynamique très efficace pour stabiliser les patients avec une EP grave compliquée d'un état de choc réfractaire ou d'un arrêt cardiaque. L'augmentation de la postcharge du ventricule droit par les caillots dans les artères pulmonaires étant à l'origine de la défaillance circulatoire au cours de l'EP grave, il semble logique d'y associer une procédure de désobstruction afin de lever rapidement l'obstruction à l'éjection du ventricule droit sans attendre l'effet de la fibrinolyse physiologique qui peut prendre plusieurs jours sous anticoagulation efficace. L'efficacité et surtout la sécurité de l'ECMO seule ou en association avec une procédure de désobstruction nécessitent des études cliniques prospectives dédiées. Ces patients étant rares, il est vraisemblablement nécessaire de prendre l'avis d'équipes référentes pluridisciplinaires.

\section{Questions en suspens}

Chez les patients les plus graves avec un état de choc réfractaire, la place de l'assistance circulatoire extracorporelle seule ou en association à des traitements de reperfusion vasculaire pulmonaire (fibrinolyse systémique, thrombectomie percutanée dirigée par cathéter ou chirurgicale) par rapport à l'embolectomie chirurgicale n'est pas clairement établie. Les techniques de thrombectomie percutanée nécessitent une évaluation dans des essais randomisés dédiés. Enfin, l'intérêt d'une dose plus faible de thrombolytique chez les patients avec une EP à risque intermédiaire élevé reste à évaluer.

\section{Conclusion}

La stratification du risque individuel de mortalité précoce permet d'apporter une stratégie diagnostique et thérapeutique optimisée pour chaque patient. Les techniques de revascularisation doivent être systématiquement proposées chez les patients à risque élevé et chez les patients à risque intermédiaire haut présentant une dégradation hémodynamique secondaire (Tableau 3). Le positionnement dans l'algorithme thérapeutique de l'assistance extracorporelle et des traitements percutanés reste à définir. Leurs indications doivent donc être posées dans des centres experts et après une concertation multidisciplinaire incluant pneumologues, cardiologues, réanimateurs, radiologues interventionnels et chirurgiens cardiaques.

Liens d'intérêts : Gilles Meyer déclare avoir reçu des fonds de recherche des laboratoires LEO Pharma, Bayer Healthcare et BMS-Pfizer ainsi que des invitations en congrès des laboratoires LEO Pharma, Daiichi-Sankyo et BMS-Pfizer.

Olivier Sanchez déclare avoir fait partie du board du laboratoire BTG.

Les autres auteurs déclarent ne pas avoir de lien d'intérêt.

\section{Références}

1. Cohen AT, Agnelli G, Anderson FA, Arcelus JI, Bergqvist D, Brecht JG, Greer IA, Heit JA, Hutchinson JL, Kakkar AK, Mottier D, Oger E, Samama MM, Spannag M; (VITAE) for the VIAG in E, (2007) Venous thromboembolism (VTE) in Europe the number of VTE events and associated morbidity and mortality. Thromb Haemost 98: 756-764 
2. L'état de santé de la population en France. Rapport 2017. http:// invs.santepubliquefrance.fr/publications/etat_sante_2017/ESP2017_ Ouvrage_complet_vdef.pdf

3. Konstantinides SV, Torbicki A, Agnelli G, Danchin N, Fitzmaurice D, Galiè N, Gibbs JSR, Huisman MV, Humbert M, Kucher N, Lang I, Lankeit M, Lekakis J, Maack C, Mayer E, Meneveau N, Perrier A, Pruszczyk P, Rasmussen LH, Schindler TH, Svitil P, Noordegraaf AV, Zamorano JL, Zompatori M, Achenbach S, Baumgartner H, Bax JJ, Bueno H, Dean V, Deaton C, Erol Ç, Fagard R, Ferrari R, Hasdai D, Hoes A, Kirchhof P, Knuuti J, Kolh P, Lancellotti P, Linhart A, Nihoyannopoulos P, Piepoli MF, Ponikowski P, Sirnes PA, Tamargo JL, Tendera M, Wijns W, Windecker S, Jimenez D, Ageno W, Agewall S, Asteggiano R, Bauersachs R, Becattini C, Bounameaux H, Büller HR, Davos CH, Geersing GJ, Sanchez MAG, Hendriks J, Kilickap M, Mareev V, Monreal M, Morais J, Popescu BA, Sanchez O, Spyropoulos AC, (2014) 2014 ESC Guidelines on the diagnosis and management of acute pulmonary embolism. Eur Heart J 35: 3033-3080

4. Kasper W, Konstantinides S, Geibel A, Olschewski M, Heinrich F, Grosser KD, Rauber K, Iversen S, Redecker M, Kienast J, (1997) Management strategies and determinants of outcome in acute major pulmonary embolism: results of a multicenter registry. J Am Coll Cardiol 30: 1165-1173

5. Goldhaber SZ, Visani L, De Rosa M, (1999) Acute pulmonary embolism: clinical outcomes in the International Cooperative Pulmonary Embolism Registry (ICOPER) [see comment]. Lancet 353: 1386-1389

6. Laporte S, Mismetti P, Décousus H, Uresandi F, Otero R, Lobo JL, Monreal M, the RIETE Investigators, (2008) Clinical predictors for fatal pulmonary embolism in 15,520 patients with venous thromboembolism: findings from the Registro Informatizado de la Enfermedad TromboEmbolica venosa (RIETE) registry. Circulation 117: 1711-1716

7. Sanchez O, Trinquart L, Caille V, Couturaud F, Pacouret G, Meneveau N, Verschuren F, Roy PM, Parent F, Righini M, Perrier A, Lorut C, Tardy B, Benoit MO, Chatellier G, Meyer G, (2010) Prognostic factors for pulmonary embolism: the PREP study, a prospective multicenter cohort study. Am J Respir Crit Care Med 181: 168-173

8. Aujesky D, Obrosky DS, Stone RA, Auble TE, Perrier A, Cornuz J, Roy PM, Fine MJ, (2005) Derivation and validation of a prognostic model for pulmonary embolism. Am J Respir Crit Care Med 172: 1041-1046

9. Jimenez D, Aujesky D, Moores L, Gomez V, Lobo JL, Uresandi F, Otero R, Monreal M, Muriel A, Yusen RD, (2010) Simplification of the pulmonary embolism severity index for prognostication in patients with acute symptomatic pulmonary embolism. Arch Intern Med 170: 1383-1389

10. Righini M, Roy PM, Meyer G, Verschuren F, Aujesky D, Le Gal G, (2011) The Simplified Pulmonary Embolism Severity Index (PESI): validation of a clinical prognostic model for pulmonary embolism. J Thromb Haemost JTH 9: 2115-7

11. Kohn CG, Mearns ES, Parker MW, Hernandez AV, Coleman CI, (2015) Prognostic accuracy of clinical prediction rules for early post-pulmonary embolism all-cause mortality: a bivariate metaanalysis. Chest 147: 1043-1062

12. Elias A, Mallett S, Daoud-Elias M, Poggi JN, Clarke M, (2016) Prognostic models in acute pulmonary embolism: a systematic review and meta-analysis. BMJ Open 6: e010324

13. Hellenkamp K, Schwung J, Rossmann H, Kaeberich A, Wachter R, Hasenfuß G, Konstantinides S, Lankeit M, (2015) Risk stratification of normotensive pulmonary embolism: prognostic impact of copeptin. Eur Respir J 46: 1701-1710
14. Bova C, Sanchez O, Prandoni P, Lankeit M, Konstantinides S, Vanni S, Jiménez D, (2014) Identification of intermediate-risk patients with acute symptomatic pulmonary embolism. Eur Respir J 44: 694-703

15. Jiménez D, Kopecna D, Tapson V, Briese B, Schreiber D, Lobo J, Monreal M, Aujesky D, Sanchez O, Meyer G, Konstantinides S, Yusen RD, (2014) Derivation and validation of multimarker prognostication for normotensive patients with acute symptomatic pulmonary embolism. Am J Respir Crit Care Med 189: 718-726

16. Becattini C, Agnelli G, Lankeit M, Masotti L, Pruszczyk P, Casazza F, Vanni S, Nitti C, Kamphuisen P, Vedovati MC, De Natale MG, Konstantinides S, (2016) Acute pulmonary embolism: mortality prediction by the 2014 European Society of Cardiology risk stratification model. Eur Respir J 48: 780-786

17. Lankeit M, Jiménez D, Kostrubiec M, Dellas C, Kuhnert K, Hasenfuß G, Pruszczyk P, Konstantinides S, (2014) Validation of $\mathrm{N}$-terminal pro-brain natriuretic peptide cut-off values for risk stratification of pulmonary embolism. Eur Respir J 43: 1669-1677

18. Ucar EY, Araz O, Akgun M, Meral M, Kalkan F, Saglam L, Kaynar H, Gorguner AM, (2013) Low-molecular-weight heparin use with thrombolysis: is it effective and safe? Ten years' clinical experience. Respir Int Rev Thorac Dis 86: 318-323

19. Sharifi M, Bay C, Skrocki L, Rahimi F, Mehdipour M, (2013) Moderate Pulmonary Embolism Treated With Thrombolysis (from the "MOPETT" Trial). Am J Cardiol 111: 273-277

20. Hao Q, Dong BR, Yue J, Wu T, Liu GJ, (2015) Thrombolytic therapy for pulmonary embolism. Cochrane Database Syst Rev CD004437

21. Chatterjee S, Chakraborty A, Weinberg I, Kadakia M, Wilensky RL, Sardar P, Kumbhani DJ, Mukherjee D, Jaff MR, Giri J, (2014) Thrombolysis for pulmonary embolism and risk of allcause mortality, major bleeding, and intracranial haemorrhage: a meta-analysis. JAMA 311: 2414

22. Marti C, John G, Konstantinides S, Combescure C, Sanchez O, Lankeit M, Meyer G, Perrier A, (2015) Systemic thrombolytic therapy for acute pulmonary embolism: a systematic review and meta-analysis. Eur Heart J 36: 605-614

23. Nakamura S, Takano H, Kubota Y, Asai K, Shimizu W, (2014) Impact of the efficacy of thrombolytic therapy on the mortality of patients with acute submassive pulmonary embolism: a metaanalysis. J Thromb Haemost JTH 12: 1086-1095

24. Wang TF, Squizzato A, Dentali F, Ageno W, (2015) The role of thrombolytic therapy in pulmonary embolism. Blood 125: 21912199

25. Riera-Mestre A, Becattini C, Giustozzi M, Agnelli G, (2014) Thrombolysis in hemodynamically stable patients with acute pulmonary embolism: a meta-analysis. Thromb Res 134: 1265-1271

26. Meyer G, Vicaut E, Danays T, Agnelli G, Becattini C, BeyerWestendorf J, Bluhmki E, Bouvaist H, Brenner B, Couturaud F, Dellas C, Empen K, Franca A, Galiè N, Geibel A, Goldhaber SZ, Jimenez D, Kozak M, Kupatt C, Kucher N, Lang IM, Lankeit M, Meneveau N, Pacouret G, Palazzini M, Petris A, Pruszczyk P, Rugolotto M, Salvi A, Schellong S, Sebbane M, Sobkowicz B, Stefanovic BS, Thiele H, Torbicki A, Verschuren F, Konstantinides SV, (2014) Fibrinolysis for patients with intermediate-risk pulmonary embolism. N Engl J Med 370: 1402-1411

27. Konstantinides SV, Vicaut E, Danays T, Becattini C, Bertoletti L, Beyer-Westendorf J, Bouvaist H, Couturaud F, Dellas C, Duerschmied D, Empen K, Ferrari E, Galiè N, Jiménez D, Kostrubiec M, Kozak M, Kupatt C, Lang IM, Lankeit M, Meneveau N, Palazzini M, Pruszczyk P, Rugolotto M, Salvi A, Sanchez O, Schellong S, Sobkowicz B, Meyer G, (2017) Impact of thrombolytic therapy on the long-term outcome of intermediate-risk pulmonary embolism. J Am Coll Cardiol 69: 1536-1544 
28. Wang C, Zhai Z, Yang Y, Wu Q, Cheng Z, Liang L, Dai H, Huang K, Lu W, Zhang Z, (2010) Efficacy and safety of low dose recombinant tissue-type plasminogen activator for the treatment of acute pulmonary thromboembolism: a randomized, multicenter, controlled trial. Chest J 137: 254-262

29. Kucher N, Boekstegers P, Muller OJ, Kupatt C, BeyerWestendorf J, Heitzer T, Tebbe U, Horstkotte J, Muller R, Blessing E, Greif M, Lange P, Hoffmann RT, Werth S, Barmeyer A, Hartel D, Grunwald H, Empen K, Baumgartner I, (2014) Randomized, controlled trial of ultrasound-assisted catheter-directed thrombolysis for acute intermediate-risk pulmonary embolism. Circulation 129: 479-486

30. Bajaj NS, Kalra R, Arora P, Ather S, Guichard JL, Lancaster WJ, Patel N, Raman F, Arora G, Al Solaiman F, Clark DT, Dell'Italia LJ, Leesar MA, Davies JE, McGiffin DC, Ahmed MI, (2016) Catheter-directed treatment for acute pulmonary embolism: systematic review and single-arm meta-analyses. Int $\mathrm{J}$ Cardiol 225: $128-139$

31. Du GC, Zhang MC, Zhao JC, (2015) Catheter-directed thrombolysis plus anticoagulation versus anticoagulation alone in the treatment of proximal deep vein thrombosis: a meta-analysis. VASA Z Gefasskrankheiten 44: 195-202

32. Kuo WT, Gould MK, Louie JD, Rosenberg JK, Sze DY, Hofmann LV, (2009) Catheter-directed therapy for the treatment of massive pulmonary embolism: systematic review and metaanalysis of modern techniques. J Vasc Interv Radiol JVIR 20: $1431-1440$

33. Mostafa A, Briasoulis A, Shokr M, Briasouli AA, Panaich S, Grines C, (2016) Ultrasound accelerated thrombolysis in patients with acute pulmonary embolism: a systematic review and proportion meta-analysis. Int J Cardiol 211: 27-30

34. Kuo WT, Banerjee A, Kim PS, DeMarco FJ, Levy JR, Facchini FR, Unver K, Bertini MJ, Sista AK, Hall MJ, (2015) Pulmonary embolism response to fragmentation, embolectomy, and catheter thrombolysis (PERFECT): initial results from a prospective multicenter registry. Chest J 148: 667-673

35. Piazza G, Hohlfelder B, Jaff MR, Ouriel K, Engelhardt TC, Sterling KM, Jones NJ, Gurley JC, Bhatheja R, Kennedy RJ, Goswami N, Natarajan K, Rundback J, Sadiq IR, Liu SK, Bhalla N, Raja ML, Weinstock BS, Cynamon J, Elmasri FF, Garcia MJ, Kumar M, Ayerdi J, Soukas P, Kuo W, Liu PY, Goldhaber SZ, SEATTLE II Investigators, (2015) A prospective, single-arm, multicenter trial of ultrasound-facilitated, catheter-directed, lowdose fibrinolysis for acute massive and submassive pulmonary embolism: the SEATTLE II Study. JACC Cardiovasc Interv 8: 1382-1392

36. Myers PO, Bounameaux H, Panos A, Lerch R, Kalangos A, (2010) Impending paradoxical embolism: systematic review of prognostic factors and treatment. Chest 137: 164-170

37. Mathew TC, Ramsaran EK, Aragam JR, (1995) Impending paradoxic embolism in acute pulmonary embolism: diagnosis by transesophageal echocardiography and treatment by emergent surgery. Am Heart J 129: 826-827

38. Edelman JJ, Okiwelu N, Anvardeen K, Joshi P, Murphy B, Sanders LH, Newman MA, Passage J, (2016) Surgical pulmonary embolectomy: experience in a series of 37 consecutive cases. Heart Lung Circ 25: 1240-1244

39. Neely RC, Byrne JG, Gosev I, Cohn LH, Javed Q, Rawn JD, Goldhaber SZ, Piazza G, Aranki SF, Shekar PS, Leacche M,
(2015) Surgical embolectomy for acute massive and submassive pulmonary embolism in a series of 115 patients. Ann Thorac Surg 100: 1245-1251; (discussion 1251-1252)

40. Yoo JW, Choi HC, Lee SJ, Cho YJ, Lee JD, Kim HC, (2016) Comparison between systemic and catheter thrombolysis in patients with pulmonary embolism. Am J Emerg Med 34: 985-988

41. Cho YH, Sung K, Kim WS, Jeong DS, Lee YT, Park PW, Kim DK, (2016) Management of acute massive pulmonary embolism: is surgical embolectomy inferior to thrombolysis? Int J Cardiol 203: 579-583

42. Greelish JP, Leacche M, Solenkova NS, Ahmad RM, Byrne JG, (2011) Improved midterm outcomes for type A (central) pulmonary emboli treated surgically. J Thorac Cardiovasc Surg 142: 1423-1429

43. Vohra HA, Whistance RN, Mattam K, Kaarne M, Haw MP, Barlow CW, Tsang GMK, Livesey SA, Ohri SK, (2010) Early and late clinical outcomes of pulmonary embolectomy for acute massive pulmonary embolism. Ann Thorac Surg 90: 1747-1752

44. Meneveau N, Seronde MF, Blonde MC, Legalery P, Didier-Petit K, Briand F, Caulfield F, Schiele F, Bernard Y, Bassand JP, (2006) Management of unsuccessful thrombolysis in acute massive pulmonary embolism. Chest 129: 1043-1050

45. Aklog L, Williams CS, Byrne JG, Goldhaber SZ, (2002) Acute pulmonary embolectomy: a contemporary approach. Circulation 105: $1416-1419$

46. Mercat A, Diehl JL, Meyer G, Teboul JL, Sors H, (1999) Hemodynamic effects of fluid loading in acute massive pulmonary embolism. Crit Care Med 27: 540-544

47. Pinsky MR, (2016) The right ventricle: interaction with the pulmonary circulation. Crit Care doi: 10.1186/s13054-016-1440-0

48. Ternacle J, Gallet R, Mekontso-Dessap A, Meyer G, Maitre B, Bensaid A, Jurzak P, Gueret P, Dubois Randé JL, Lim P, (2013) Diuretics in normotensive patients with acute pulmonary embolism and right ventricular dilatation. Circ J Off J Jpn Circ Soc 77: 2612-2618

49. Meyer G, Vieillard-Baron A, Planquette B, (2016) Recent advances in the management of pulmonary embolism: focus on the critically ill patients. Ann Intensive Care 6: 19

50. Ghignone M, Girling L, Prewitt RM, (1984) Volume expansion versus norepinephrine in treatment of a low cardiac output complicating an acute increase in right ventricular afterload in dogs. Anesthesiology 60: 132-135

51. Kerbaul F, Gariboldi V, Giorgi R, Mekkaoui C, Guieu R, Fesler P, Gouin F, Brimioulle S, Collart F, (2007) Effects of levosimendan on acute pulmonary embolism-induced right ventricular failure. Crit Care Med 35: 1948-1954

52. Jardin F, Farcot JC, Boisante L, Curien N, Margairaz A, Bourdarias JP, (1981) Influence of positive end-expiratory pressure on left ventricular performance. N Engl J Med 304: 387-392

53. Szold O, Khoury W, Biderman P, Klausner JM, Halpern P, Weinbroum AA, (2006) Inhaled nitric oxide improves pulmonary functions following massive pulmonary embolism: a report of four patients and review of the literature. Lung 184: 1-5

54. Corsi F, Lebreton G, Bréchot N, Hekimian G, Nieszkowska A, Trouillet JL, Luyt CE, Leprince P, Chastre J, Combes A, Schmidt M, (2017) Life-threatening massive pulmonary embolism rescued by venoarterial-extracorporeal membrane oxygenation. Crit Care. doi: $10.1186 / \mathrm{s} 13054-017-1655-8$ 